\title{
évaluation des risques de rupture sur un tracé de remblai sur sols mous
}

\author{
estimation of the risk of failure on a project of embankment \\ on soft soils
}

\author{
J.-P. MAGNAN \\ Chef de la Division de Géotechnique-Mécanique des Sols $1^{*}$ \\ C. MAHDAVI \\ Docteur-Ingénieur"
}

Rev. Franç. Géotech. n 44, p.p. $43-55$ (juillet 1988)

\section{Résumé}

Les variations des propriétés des sols le long des tracés sont une source d'incertitude et donc de risque pour la stabilité des ouvrages. Cet article décrit la procédure utilisée pour évaluer ce risque sur un tracé de remblai sur sols mous. L'analyse des risques de rupture est réalisée en deux temps. On analyse d'abord les variations des valeurs des propriétés des sols servant au calcul, en utilisant des corrélations et la technique d'interpolation statistique par krigeage développée en géostatistique. Puis on étudie, par la méthode de Monte Carlo, la distribution des coefficients de sécurité calculés dans l'hypothèse de la rupture circulaire de blocs de longueur finie. Cette procédure permet de déterminer la position et la longueur des zones de plus grand risque le long du tracé.

\footnotetext{
Abstract

The spatial variations of soil properties along a project line are a source of uncertainty, thus of risk, with respect to the stability of structures. This paper describes a procedure for estimating this risk in the case of an embankment on soft soils. The variations of the soil characteristics used for the design are first analyzed, using correlations and the technique of kriging developed in geostatistics. The Monte Carlo method is then used for determining the distribution of the safety factors for circular failure of blocks of limited length. By using this procedure, the location and length of the zones of highest risk can be estimated.
} 


\section{INTRODUCTION}

L'analyse de la stabilité des remblais sur sols mous pose peu de problèmes méthodologiques à l'heure actuelle (MAGNAN et al., 1984. LEROUEIL et al., 1985). L'utilisation des cohésions non drainées mesurées au scissomètre, corrigées en fonction de la plasticité du sol selon la procédure popularisée par BJERRUM (1972), dans des calculs en rupture circulaire, permet de fixer avec précision les hauteurs de remblais que l'on peut mettre en place sans risque de rupture. Dans la pratique française, des coefficients de sécurité de 1,5 sont normalement utilisés, sans que ce choix s'appuie sur une évaluation de probabilité de rupture; on sait seulement qu'il permet d'éviter les ruptures si les études géotechniques sont bien menées, tandis que des valeurs plus faibles avaient été utilisées dans les cas où des désordres ou des ruptures s'étaient produits.

L'idée de caractériser les risques de rupture des remblais sur sols mous par une probabilité de rupture, et non par un coefficient de sécurité à seuil empirique, est apparue au cours des années 1970, à une époque où différents chercheurs ont tenté d'harmoniser les valeurs des coefficients de sécurité de la mécanique des sols en comparant les probabilités de ruine associées. MAGNAN et BAGHERY (1982) ont passé en revue seize articles consacrés à l'analyse probabiliste de la stabilité des remblais et des pentes au cours de cette décennie et testé sur l'un des remblais du site expérimental de Cubzac-les-Ponts la méthode décrite par ALONSO (1976). Dix autres publications ont été analysées par MAHDAVI (1985). Ces études ont montré (BAGHERY, 1980; BAGHERY et MAGNAN, 1983) que la réalisation matérielle de calculs probabilistes était possible, à condition de disposer de nombreuses données sur les propriétés des sols, mais que les résultats en termes de probabilités de rupture dépendaient beaucoup (trop) des hypothèses arbitraires qu'il faut faire sur les distributions de probabilités des coefficients de sécurité calculés. De plus, l'approche probabiliste était à l'évidence incapable de corriger les effets des erreurs systématiques dans l'estimation des paramètres de calcul, comme celle qui a motivé la correction de BJERRUM en fonction de la plasticité. Tous ces faits nous ont conduits à renoncer provisoirement à appliquer cette méthode d'analyse à d’autres exemples.

L'étude présentée ici a été entreprise peu après la fin des travaux précédents, pour évaluer la possibilité de réaliser des études de risques le long de tracés de remblais sur sols mous. Les ingénieurs chargés de préparer les projets de routes disposent en général d'informations peu denses sur les propriétés des sols le long du tracé à étudier. Quelques dizaines de mètres sont une distance minimale entre les sondages et il leur faut imaginer les propriétés des sols entre les points de mesure. Il existe donc un risque que la portance du sol soit plus faible entre les sondages. Nous avons tenté d'évaluer ce risque en utilisant les outils de la géostatistique. La méthode d'analyse a été appliquée aux données recueillies lors de l'étude géotechnique de l'un des tracés de l'autoroute A 64 dans la basse vallée de l'Adour.

\section{PROCÉDURE DE CALCUL}

L'analyse probabiliste des ouvrages comporte en pratique deux phases successives de traitement statistique

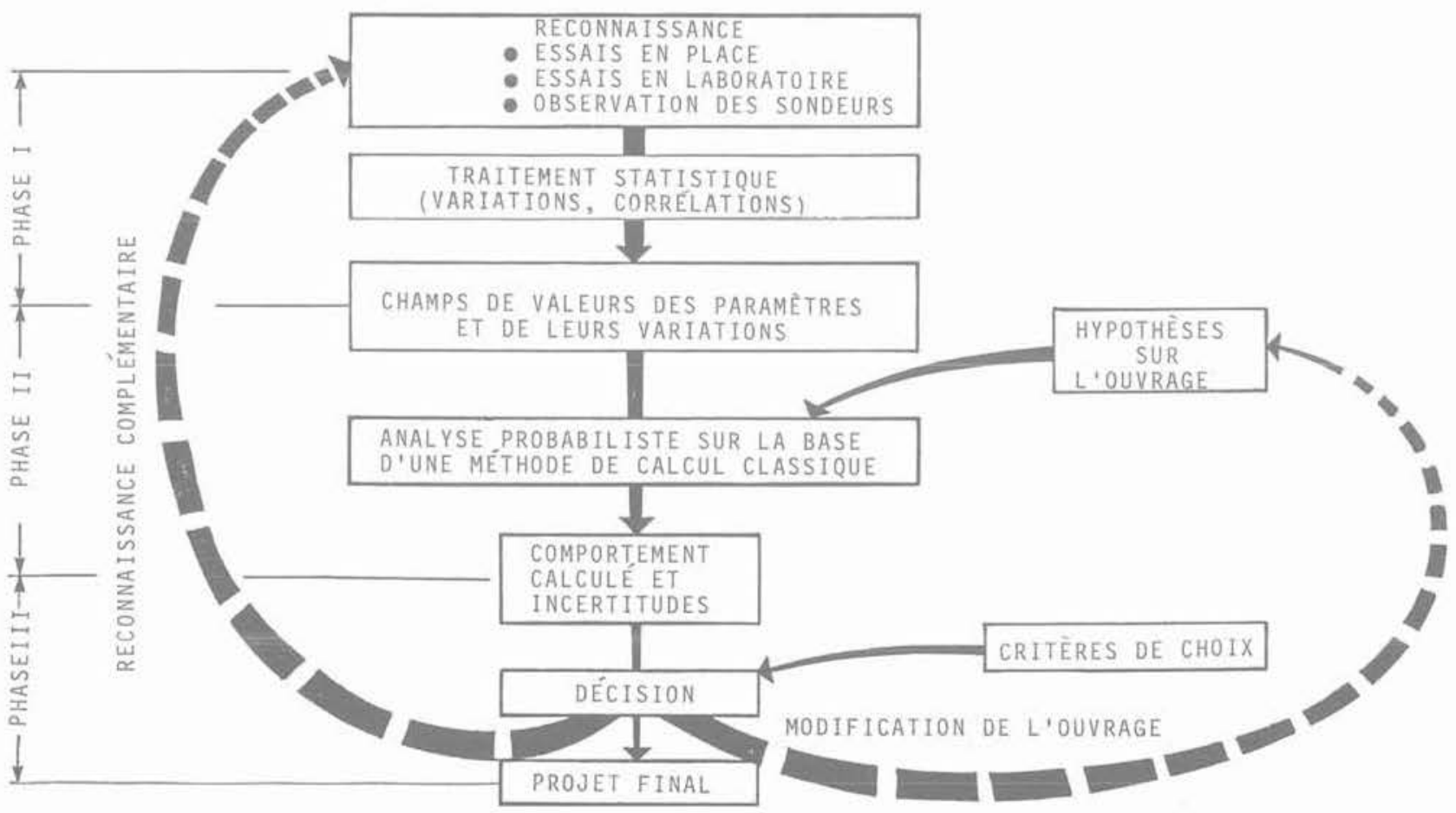

Fig. 1, - Phases du dimensionnement probabiliste d'un ouvrage.

Fig. 1. - Stages of probabilistic design of a structure. 
des données puis de calcul probabiliste, suivies d'une phase d'examen des résultats et de décision (fig. 1). Le choix de linterface entre les deux phases d'analyse, c'est-à-dire de la forme des données géotechniques qui serviront au calcul de louvrage, a une importance primordiale pour la réussite de l'étude. Le champ des valeurs moyennes estimées de chaque propriété dans le massif de sol et le champ des erreurs d'estimation de ces mêmes propriétés en chaque point semblent constituer une bonne base de travail, dans l'état actuel de nos techniques d'analyse.

\subsection{Analyse statistique des variations spatiales des propriétés des sols (Phase 1)}

La procédure adoptée pour l'analyse des variations spatiales des propriétés des sols dans un massif est analogue dans son principe à la démarche de l'ingé. nieur qui cherche à tirer le meilleur parti possible des informations disponibles pour un projet: après la réa. lisation des sondages, essais en place et essais de laboratoire (fig. 2), cet ingénieur essaie de diviser le massif en ensembles homogènes du point de vue:

- de la nature du sol (qui définit ses principes de comportement),

- de ses propriétés physiques et mécaniques,

- de son état.

Lorsqu'il dispose d'un nombre insuffisant de valeurs de certains des paramètres, il peut utiliser des corrélations pour compléter sa description du milieu naturel. C'est souvent le cas dans les projets courants, où l'on a tendance à multiplier les essais en place rapides au détriment des essais mécaniques plus lents et onéreux et où la densité globale des essais reste faible (MA. GNAN et al., 1980, conseillent par exemple des densités d'essais en place et de sondages de l'ordre de trente au kilomètre, dans le cas des tracés, et de vingt pour

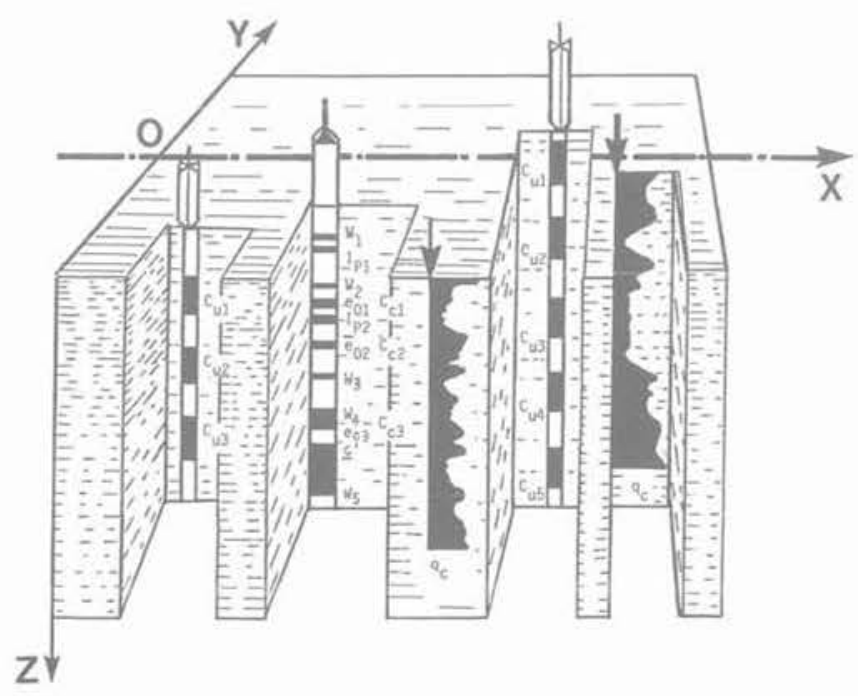

Fig. 2. - Résultats bruts de la reconnaissance géotechnique d'un site.

Fig. 2. - Untreated results of the geotechnical reconnaissance of a site. vingt-cinq hectares, dans le cas de zones de sols compressibles). L'ingénieur attribue ensuite des valeurs aux paramètres de calcul dans chacun de ces ensembles homogènes.

La procédure que nous avons adoptée comporte les étapes suivantes :

1. Etablissement de cartes des valeurs mesurées de chaque paramètre:

2. Définition de couches homogènes du point de vue de la géotechnique;

3. Etude des corrélations entre paramètres à l'intérieur de chaque couche;

4. Etablissement de la carte des points où au moins un paramètre a été mesuré;

5. Calcul en chacun de ces points de la valeur des paramètres non mesurés, en utilisant les corrélations ;

6. Etablissement, pour chaque paramètre, d'une carte des valeurs mesurées ou estimées et d'une carte des incertitudes sur ces valeurs (dues aux erreurs de mesure ou à l'utilisation des corrélations) :

7. Etude de la distribution des incertitudes et de l'influence de la donnée d'informations complémentaires dans les zones où l'incertitude est maximale (ce qui permet d'orienter le choix de reconnaissances complémentaires éventuelles);

8. Compte tenu de la méthode d'analyse probabiliste à utiliser, génération des champs de valeurs de calcul des paramètres géotechniques du sol pour des coupes transversales ou longitudinales quelconques.

Sans entrer ici dans le détail des procédures de calcul, on peut indiquer que les corrélations entre paramètres servent uniquement à calculer les valeurs manquantes des paramètres de calcul et l'incertitude associée, et que toutes les méthodes d'analyse conduisant à un résultat explicite (de la forme $\mathrm{Y}=\mathrm{f}[\mathrm{X}]$ ) peuvent être utilisées. Lorsque l'on ne dispose pas de données assez nombreuses pour établir des corrélations locales sur le site du projet, on peut naturellement utiliser des corrélations établies sur d'autres sites, mais l'erreur d'estimation s'en trouve généralement augmentée.

Pour l'interpolation des valeurs mesurées ou estimées par corrélations et le calcul des paramètres géotechniques aux points nécessaires pour le calcul, on utilise les procédures de krigeage développées en géostatistique, l'incertitude sur les valeurs estimées (et éventuellement les erreurs de mesure) étant introduites dans les termes diagonaux des matrices à inverser (voir, par exemple, GUILLAUME, 1977, ou MAGNAN, 1982).

La représentation graphique des champs de valeurs moyennes estimées et des incertitudes (variances ou écarts types) est généralement complexe: pour les paramètres définis dans un espace bidimensionnel (coupe transversale ou longitudinale de l'ouvrage), on peut adopter la représentation de la figure 3 , à condition de représenter de façon continue les variations selon une direction et de façon discontinue les variations selon d'autre direction. On peut également donner des tableaux de valeurs aux points servant au calcul, ce qui est moins expressif. 


\subsection{Analyse probabiliste de la stabilité d'un remblai sur sol mou (Phase 2)}

La procédure de calcul des ouvrages, sur la base d'un champ de valeurs probables des paramètres de calcul en chaque point de l'espace et de leur incertitude, est conditionnée par le schéma de calcul déterministe que l'on choisit. Elle est de ce fait différente d'un type d'ouvrage à l'autre et, pour un ouvrage donné, d'une méthode de calcul à l'autre, même si le mode de raisonnement est le même dans tous les cas.

Pour le problème que nous avons étudié, celui de l'incertitude sur la stabilité d'un remblai de très grande longueur construit sur des sols mous, nous avons adopté un schéma de calcul très simple: rupture par rotation d'un cylindre de sol limité par deux surfaces planes. Ce choix, a priori un peu simpliste, a été fait pour plusieurs raisons:

- un des objectifs principaux de notre travail était de chercher à quantifier le risque que l'on prend quand on dimensionne un remblai de très grande longueur sur la base de données géotechniques mesurées seulement en certains points du tracé. La longueur de la zone de rupture dans les remblais sur sols mous peut être plus ou moins grande, mais elle est toujours limitée, ce qui rendait nécessaire d'abandonner le modèle de calcul classique " en rupture circulaire " pour un modèle de calcul où le volume de sol et de remblai concerné par le glissement est limité;
- comme on ne connait pas a priori la longueur de la zone de rupture, qui dépend de la longueur sur laquelle la résistance du sol est plus faible et de l'in. certitude sur les valeurs estimées de cette résistance, et comme on ne peut faire le calcul de stabilité qu'en testant l'équilibre de cylindres d'axes et de diamètres variés, on savait par avance qu'il faudrait réaliser un très grand nombre de calculs sur ordinateur. Il a paru de ce fait préférable d'adopter un schéma de calcul simple pour limiter le coût des calculs;

- la structure spatiale des données géotechniques disponibles sur le site où l'on souhaitait appliquer la méthodologie développée ici a conduit d'autre part à admettre que la résistance du sol variait en profondeur par couches d'épaisseur constante, qu'elle ne dépendait pas de la distance horizontale à l'axe du remblai et qu'elle restait constante par tronçons de $50 \mathrm{~m}$ le long de l'axe du remblai. Ces hypothêses rendaient particulièrement intéressant le choix de surfaces de rupture cylindriques à extrémités planes, car elles permettent de séparer dans le calcul les paramètres géométriques, supposés connus sans incertitude, et les paramètres de résistance du sol, sur lesquels porte l'incertitude.

La démarche adoptée a donc consisté à :

- calculer les coefficients géométriques de toutes les surfaces de rupture envisageables, que l'on stocke dans la mémoire de l'ordinateur,

- analyser l'incertitude sur la stabilité en utilisant la méthode de Monte Carlo: génération d'un grand nombre de valeurs de la résistance du sol dans chaque

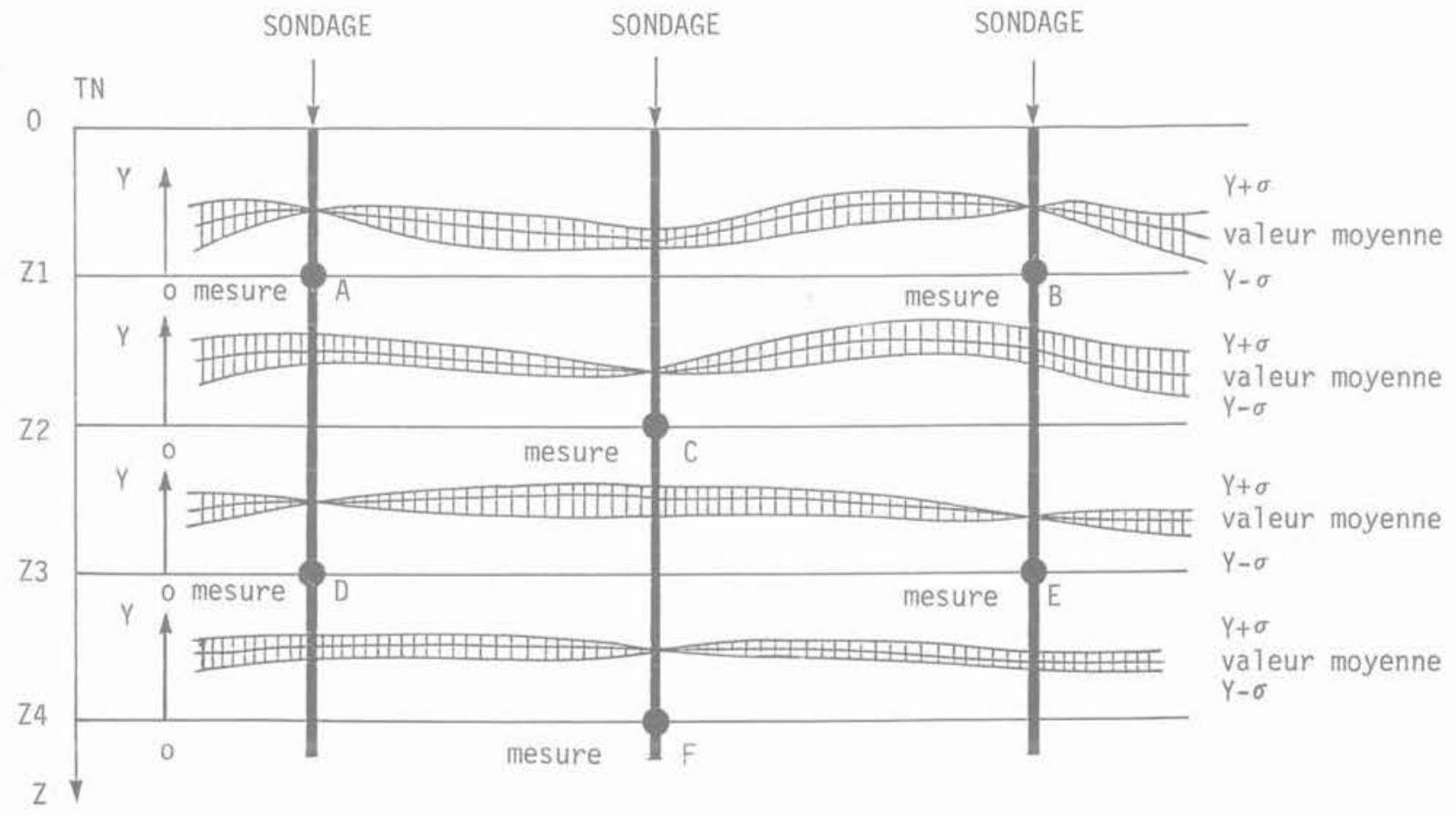

Fig. 3. - Représentation des variations spatiales estimées des propriétés d'un sol (on a supposé que les valeurs mesurées étaient exactes, sans erreur de mesure).

Fig. 3, - Representation of the estimated spatial variations of the properties of a soil (the measured values were assumed to be exact. without measurement errors) 
volume élémentaire (couche d'épaisseur connue, de longueur connue et de largueur infinie), calcul de stabilité pour chaque ensemble de valeurs de la résistance, analyse statistique des valeurs calculées du coefficient de sécurité.

\subsection{Procédure de décision (Phase 3)}

Une fois que l'on a calculé l'incertitude sur les résultats des calculs de dimensionnement de louvrage, il faut décider si l'on accepte le risque estimé, si l'on veut diminuer ce risque en diminuant le niveau de sollicitation du sol (d'où un nouveau calcul, avec une nouvelle géométrie du remblai) ou si l'on veut limiter le risque en diminuant l'incertitude sur les propriétés géotechniques des sols (d'où des essais en place ou sondages supplémentaires et la reprise des phases 1 et 2 de l'étude).

Ce processus de décision, que l'on peut formaliser en termes de la théorie de la décision, n'a pas été abordé dans cette étude.

\section{ANALYSE DES DONNÉES}

Les méthodes utilisées pour l'analyse des résultats des essais en place et en laboratoire sont simples et laissent une place à l'intervention de l'ingénieur à certains stades de la procédure, notamment pour la définition des couches " homogènes " à l'intérieur desquelles on établira des corrélations. Ce mélange de calculs automa. tiques et d'interventions humaines a été voulu dès le lancement de cette étude; il est rendu possible par la structure du système d'analyse développé à cette fin, qui s'appuie sur des logiciels existants et représente sur écran graphique les distributions des valeurs mesurées ou estimées des paramètres à chacune des étapes de lanalyse.

Le logiciel BLUEPACK-3D, développé par le Centre de Géostatistique et de Morphologie Mathématique de l'Ecole Nationale Supérieure des Mines de Paris, a été utilisé pour effectuer l'interpolation statistique par krigeage des données mesurées lors de la reconnaissance. La version dont disposait le L.C.P.C. à l'époque ne permettait pas d'effectuer des analyses par cokrigeage, en analysant simultanément les variations de deux pa. ramètres, de sorte que nous avons développé en préa. lable au krigeage une procédure d'analyse des corréla. tions entre les paramètres mesurés du sol, afin de compléter par des valeurs déduites des corrélations le champ des valeurs mesurées des paramètres utilisés pour le calcul de la stabilité du remblai.

Les procédures informatiques d'analyse des données comportent quatre étapes:

- on constitue d'abord le fichier des données expérimentales, qui comprend les informations suivantes:

- le nombre de sondages,

- l'abcisse de chaque sondage par rapport à l'origine du tracé,

- le nombre de points de mesures,

- les cotes des points de mesure et la valeur de la caractéristique mesurée,
- la position de l'axe et la profondeur maximale de chaque sondage;

- on lance ensuite l'exécution du programme P1, qui a pour rôle la visualisation des sondages et l'établissement de la carte de la reconnaissance géotechnique. $\mathrm{Ce}$ programme offre différentes options d'échelles verticales et horizontales. Il permet également de définir de façon interactive les limites des couches qui seront considérées comme homogènes pour la suite de l'analyse ;

- le programme P2 étudie d'abord les corrélations entre les valeurs des paramètres, à l'intérieur des couches définies à l'étape précédente, puis il génère les valeurs manquantes des paramètres de calcul en se servant des données disponibles et des corrélations obtenues. Ensuite, il établit la carte des valeurs mesurées ou estimées par les corrélations. Ce programme a été établi pour analyser les corrélations de deux paramètres, compte tenu de l'application envisagée: la cohésion non drainée et la résistance de pointe mesurée au pénétromètre statique (en fait, on a utilisé la valeur moyenne des résistances de pointe sur une hauteur de $8 \mathrm{~cm}$, correspondant à la hauteur du moulinet du scissomètre). Il pourrait être facilement étendu au cas

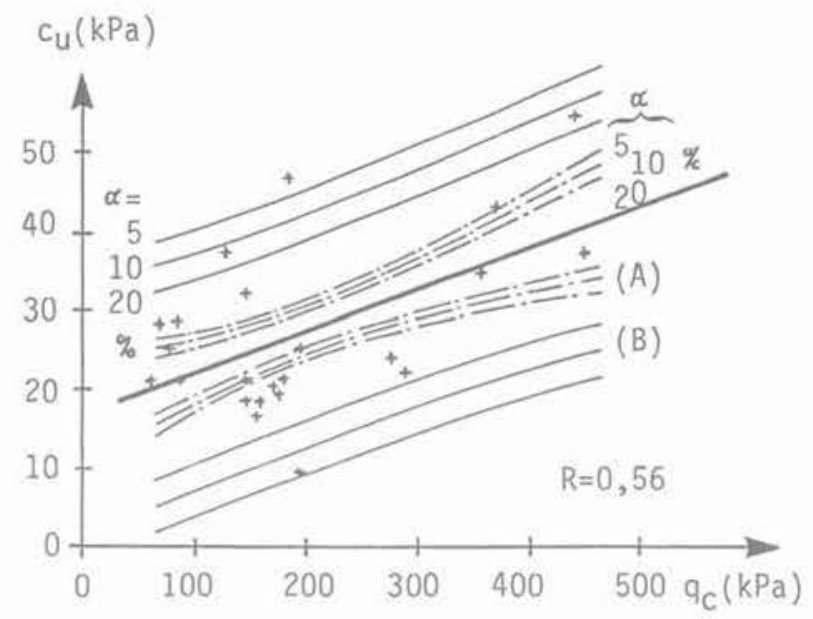

Fig. 4. - Exemple de corrélation dans une couche: (A) intervalles de confiance de $E\left[c_{u}\right]\left(q_{c}\right)$ et $(B)$ - intervalles de génération des valeurs de $c_{v}$ pour différentes valeurs de $\alpha$.

Fig. 4 - Example of correlation within a layer: (A) confidence intervals of $E\left[c_{v}\right]\left(q_{c}\right)$ and $(B)$ - intervals used for generating $c_{0}$ values for various values of $\alpha$

des relations de plusieurs paramètres. La technique d'analyse statistique utilisée est la régression linéaire simple, telle que décrite dans tous les ouvrages de référence sur les statistiques (BENJAMIN et CORNELL, 1970 ; LEBART et FÉNELON, 1973). Le calcul fournit la relation linéaire entre les deux paramètres analysés, le coefficient de corrélation, la variance des résidus et l'intervalle de confiance de l'espérance de la cohésion non drainée $E\left[c_{u}\right]$ pour une valeur donnée $\mathrm{q}_{\mathrm{c}}$ de la résistance de pointe (fig. 4). Pour la génération des valeurs de la cohésion non drainée, on a déterminé la valeur de la résistance de pointe aux points considérés, puis on a utilisé la relation linéaire pour déterminer l'espérance de la cohésion non drainée, puis on a estimé l'intervalle de confiance de cette valeur pour un 
niveau de confiance donné, comme indiqué sur la figure 5 . Ce sont ces intervalles de confiance qui ont été utilisés pour décrire les erreurs d'estimation des valeurs de la cohésion non drainée déduites des corrélations ;

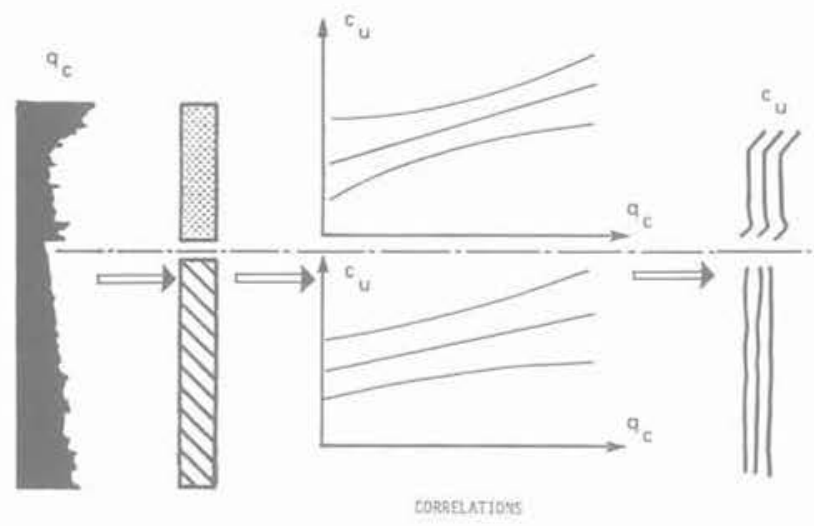

Fig. 5. - Procédure de génération d'un profil de cohésion non drainée $c_{u}$ à partir d'un profil de résistance de pointe $q_{\mathrm{c} .}$.

Fig. 5. - Procedure used for generating an undrained shear strength profile $\left(c_{u}\right)$ from a cone resistance profile $q_{c}$

- le programmes P3 est « BLUEPACK », qui est utilisé pour générer de façon systématique les profils de calcul manquants à partir des valeurs mesurées ou estimées par corrélations. La génération des valeurs de la cohésion non drainée s'effectue par krigeage ponctuel, en évaluant simultanément l'incertitude correspondant à l'estimation. Les incertitudes sont représentées en fin de calcul sous forme de cartes. Les bases théoriques de la géostatistique et du krigeage ont été décrites, par exemple, par GUILLAUME (1977), JOURNEL (1977) et DELFINER (1979). La procédure d'interpolation par krigeage comporte trois étapes principales :

- étude de la structure spatiale des variations des données et choix d'un modèle de variogramme adéquat,

- calcul des variances de l'erreur à partir de ce modèle,

- inversion du système matriciel et calcul des valeurs estimées en chaque point. Ces valeurs de la cohésion non drainée et des écarts types correspondants sont stockées dans un fichier de résultats, qui constitue l'interface de la phase d'analyse des données avec la phase d'analyse probabiliste.

\section{MÉTHODE D'ANALYSE DE LA STABILITÉ D’UN REMBLAI DE GRANDE LONGUEUR}

L'analyse des risques de rupture sur un tracé de remblai sur sols mous a été effectuée par la méthode de Monte Carlo, qui nécessite une méthode de calcul déterministe pour chacun des calculs élémentaires. La méthode de calcul déterministe utilisée pour l'analyse de la stabilité du remblai est une extension de la méthode de calcul des remblais sur sols mous à court terme, en rupture circulaire. La stabilité est analysée à court terme, en contraintes totales. Dans le sol, la résistance au cisaillement est égale à la cohésion non drainée (sans correction de plasticité, donc avec un biais qui interdit en définitive de calculer des probabilités de rupture absolues). Comme indiqué précédemment, on a choisi d'étudier la stabilité de cylindres de sol et de remblai limités par deux surfaces planes verticales (fig. 6). $\mathrm{Ce}$

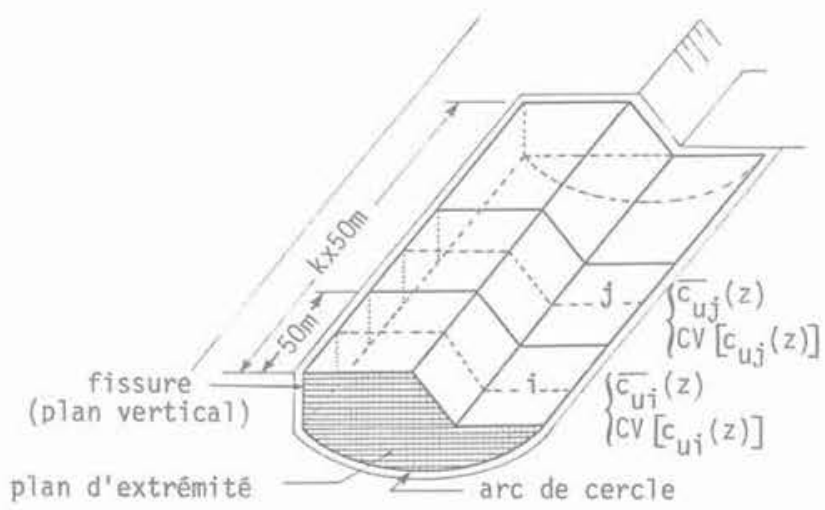

Fig. 6. - Schéma de calcul de la stabilité du remblai. Fig. 6. - Failure mechanism for the stability analysis of the fill.

choix est fidèle à l'expérience de la construction des remblais sur sols mous, qui ont toujours des ruptures d'extension limitée. Dans le remblai, on admet que le cylindre se prolonge par une fissure verticale, sur laquelle la résistance au cisaillement est nulle, ce qui simplifie les calculs. Le paramètre utilisé pour caracté. riser la stabilité du remblai est le coefficient de sécurité classique qui, dans le cas de la rupture circulaire, est le rapport du moment résistant du massif au moment moteur. Ce coefficient de sécurité est fonction de la géométrie, du poids volumique, des paramètres de résistance du massif de sol et des forces extérieures (ici. les forces de pesanteur. Le calcul et l'étude de la variation des charges appliquées au remblai n'entrent pas dans le cadre de cette étude).

Chacun de ces paramètres est affecté d'une incertitude liée au processus de mesure utilisé pour le déterminer. Les études antérieures sur la variabilité de ces paramètres ont montré que la cohésion non drainée est le paramètre qui possède la plus grande variabilité naturelle et donc la plus grande influence sur les risques de rupture. Nous avons décidé pour cette raison de considérer tous les autres paramètres de calcul comme déterministes.

S'agissant d'une étude exploratoire, nous n'avons pas cherché à mettre au point une procédure de calcul générale et la description de la résistance au cisaillement du sol est adaptée aux données disponibles sur lun des tracés de l'autoroute A 64 dans la basse vallée de l'Adour. Pour cette étude géotechnique, la maille de reconnaissance avait une structure multiple de $50 \mathrm{~m}$ le long de l'axe du tracé, de sorte que l'on ne dispose pas d'informations sur la variabilité des cohésions non drainées sur de plus faibles distances. Nous avons donc admis que la cohésion non drainée était constante à 
chaque profondeur sur chaque section de $50 \mathrm{~m}$ de longueur et que les cylindres dont la rupture serait étudiée auraient des longueurs multiples de $50 \mathrm{~m}$. Pour chaque section de $50 \mathrm{~m}$, on dispose de la valeur moyenne et du coefficient de variation de la cohésion non drainée à différentes profondeurs dans le sol. Ces valeurs ont été obtenues dans la section située au milieu de chaque tronçon, par krigeage des valeurs mesurées dans les sondages de reconnaissance géotechnique et des valeurs générées par corrélations, comme indiqué précédemment. On admit que la distance d'autocorré. lation suivant l'axe du remblai était inférieure à $50 \mathrm{~m}$. De cette façon, les valeurs obtenues par krigeage dans deux sections successives sont indépendantes. L'analyse de la stabilité s'effectuant à trois dimensions, on tient compte de la participation au moment résistant de la résistance mobilisée sur les extrémités de la surface de rupture potentielle.

Compte tenu du très grand nombre de calculs à réaliser, le calcul de stabilité a été structuré de façon particulière, afin d'éviter de recalculer de nombreuses fois les coefficients géométriques des valeurs de la cohésion non drainée: pour une géométrie donnée de la surface de nupture, on considère le moment résistant comme une fonction linéaire de toutes les valeurs de la cohésion non drainée sur la surface de rupture. Les coefficients de cette fonction linéaire dépendent uniquement de la géométrie et sont calculés une seule fois avant le début des séries de calculs de la méthode de Monte Carlo. Cette méthode d'analyse limite beaucoup la durée des calculs, mais elle demande des capacités de stockage en mémoire très importantes.

Pour l'analyse probabiliste par la méthode de Monte Carlo, on procède de la façon suivante:

- on se donne une loi de distribution du paramètre aléatoire $\left(c_{y}\right)$. Pour l'exemple présenté plus loin, une loi béta a êté utilisée :

- on génère des ensembles de valeurs aléatoires du paramètres de calcul, conformément à la loi de distribution choisie;

- on calcule, pour chaque ensemble de valeurs de la cohésion non drainée dans les différentes sous-couches du sol, le coefficient de sécurité F;

- on analyse la distribution des valeurs minimales de $F$ dans les différents calculs réalisés.

Toutes ces opérations sont réalisées par le programme P4, qui gère les opérations élémentaires d'un grand nombre de sous-programmes. Le programme $\mathrm{P} 5$ analyse les résultats des calculs réalisés par le programme P4 et les représente graphiquement, sur l'écran et sur table traçante.

Le résultat final de lanalyse probabiliste est une série de figures du type de la figure 7, qui donne la valeur de la probabilité que le coefficient de sécurité $F$ soit inférieur à un certain seuil $\beta$, pour toutes les combinaisons testées de la position et de la longueur de la zone de rupture. Chaque zone de rupture potentielle (de longueur multiple de $50 \mathrm{~m}$ ) est décrite par l'abcisse $\mathrm{x}$ de son début par rapport à l'origine du tracé et par sa longueur $\mathrm{L}_{\mathrm{c}}$.

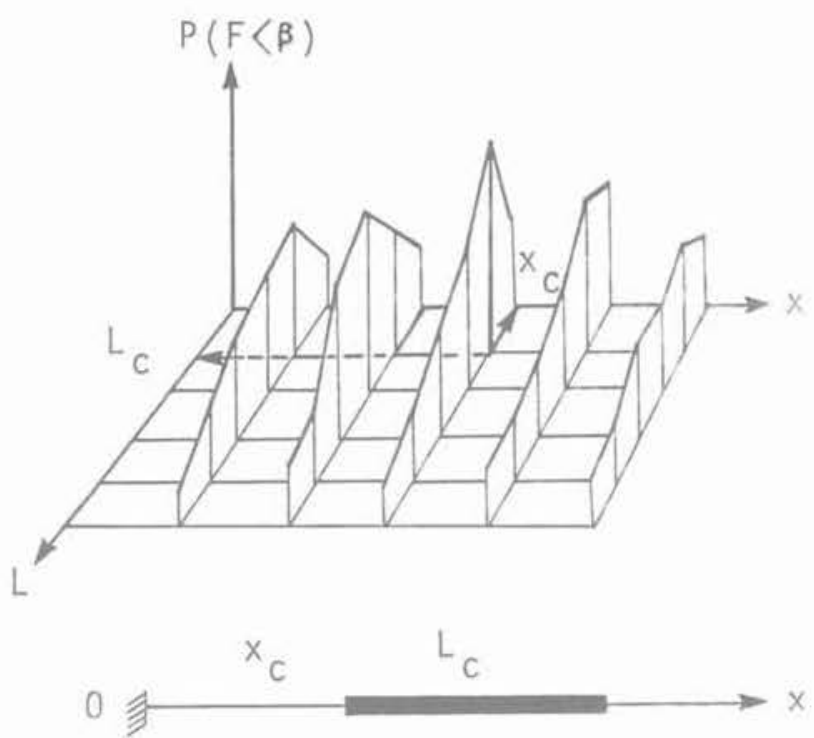

Fig. 7. - Résultat de lanalyse : probabilité que $F<p$ en fonction de la position et de la lonqueur de la zone de rupture

Fig. 7 - Result of the analysis probability of $F<\beta$ as a function of the location and length of the failed fill section

\section{APPLICATION A L'ÉTUDE D'UN TRACÉ DANS LES BARTHES DE L'ADOUR}

\subsection{Le site}

La section «Bayonne-Peyrehorade " du projet de l'Au. toroute A 64 * La Pyrénéenne * étudiée en 1978 (fig. 8) est longue de plus de $28 \mathrm{~km}$ et présente des difficultés exceptionnelles sur près de $24 \mathrm{~km}$. Les problèmes ren contrés proviennent d'une part de la traversée sur plus de $20 \mathrm{~km}$ de sols compressibles dont l'épaisseur varie de 25 à $40 \mathrm{~m}$, et d'autre part de la traversée de versants instables sur $4 \mathrm{~km}$. Pour étudier les problèmes posés par la réalisation de cette section d'autoroute, une vaste campagne de reconnaissance géotechnique a été réalisée par le Laboratoire Régional des Ponts et Chaussées de Bordeaux, avec l'appui du Laboratoire Central des Ponts et Chaussées et des Laboratoires Régionaux d'Angers, Rouen, Saint-Brieuc et Toulouse. Cette campagne de reconnaissance a comporté :

- la construction de trois remblais expérimentaux (MA GNAN et al., 1987),

- la reconnaissance sustématique de l'ensemble du tracé au moyen d'essais en place et d'essais de labo. ratoire, de façon à permettre le calcul de la stabilité et du tassement des remblais, et le choix des dispositions particulières à prévoir pour la construction.

La section analysée ici est située sur la rive gauche de l'Adour, entre les pk 18,80 et 20,70 . On peut y distin. guer quatre grandes couches de sols compressibles (fig. 9): 


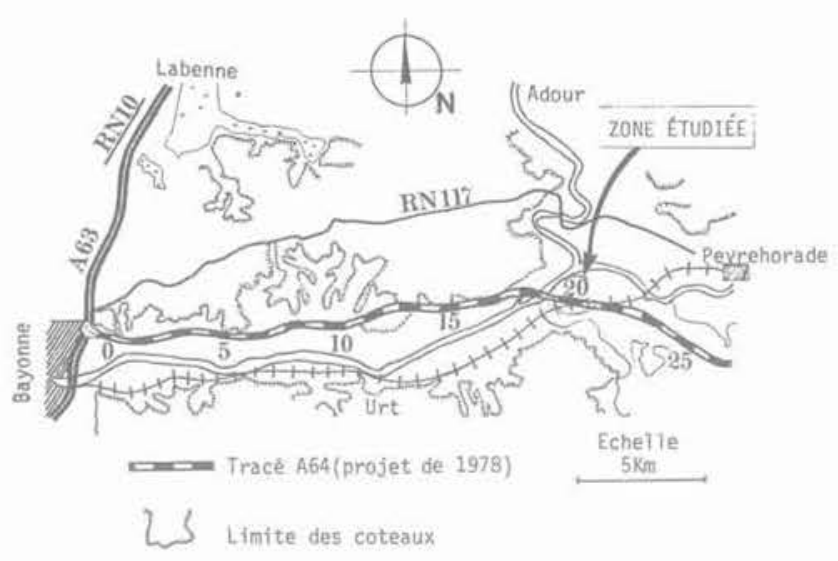

Fig. 8. - Position du tracé étudié dans la basse vallée de l'Adour.

Fig. 8. - Location of the studied embankment in the lower Adour Valley.

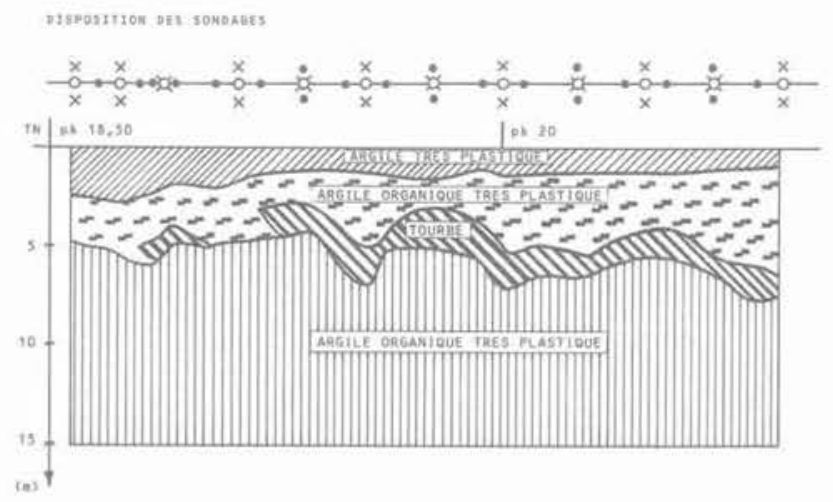

Fig. 9. - Coupe géotechnique longitudinale de la section étudiée.

Fig. 9. - Longitudinal geotechnical profile of the studied section.

- des argiles très plastiques surconsolidées en surface (1 à $2 \mathrm{~m}$ d'épaisseur),

- des limons tourbeux, localement remplacés par des sols organiques très plastiques sur 2 à $5 \mathrm{~m}$ d'épaisseur, - des tourbes marron foncé d'épaisseur comprise entre 0,3 et $2 \mathrm{~m}$,

- des sols organiques très plastiques peu consistants, sur 5 à $18 \mathrm{~m}$ d'épaisseur, suivant les endroits. Ces alluvions fines très compressibles, d'épaisseur totale voisine de $20 \mathrm{~m}$, reposent sur des terrains frottants (argiles sableuses, sables ou graves), lesquels surmontent le substratum marneux.'

La reconnaissance géotechnique a été organisée selon un maillage répétitif (fig. 10) comportant des prélèvements d'échantillons intacts au carottier à piston stationnaire sur toute l'épaisseur de la couche compressible, des essais au scissomètre de chantier et des sondages au pénétromètre statique à pointe électrique. On dispose donc d'un très grand nombre de données sur les propriétés des sols sur ce tracé, ce qui nous a permis de tester la méthode d'analyse statistique et probabiliste décrite dans ce qui précède.

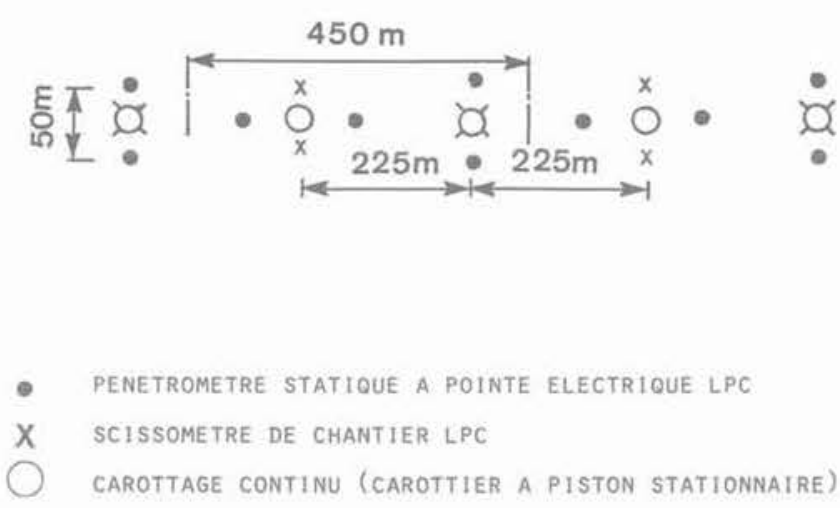

Fig. 10. - Structure de la reconnaissance géotechnique. Fig. 10. - Structure of the geotechnical reconnaissance.

\subsection{Application de la procédure d'analyse statistique}

Comme les résultats des essais au scissomètre de chantier et les sondages pénétrométriques n'étaient disponibles que sous forme écrite, la première étape de l'analyse statistique a consisté à enregistrer les résultats de 17 sondages pénétrométriques (P22 à P40) et de 13 sondages scissométriques (pour les sondages symétriques par rapport à l'axe du tracé, on a calculé la valeur moyenne des cohésions mesurées à chaque profondeur, ce qui réduit le nombre total des sondages analysés à 9), ce qui est un travail assez laborieux, notamment pour les sondages au pénétromètre.

On a ensuite procédé de la façon suivante:

- on a représenté les sondages sur l'écran du terminal graphique à une échelle de réduction convenable et on a obtenu une carte de reconnaissance géotechnique. La figure 11 montre l'apparence de l'écran (en réalité, tous les sondages sont représentés sur la même figure). On a distingué deux couches d'après les valeurs mesurées de la cohésion drainée et de la résistance de pointe, compte tenu aussi de la nature des sols (fig. 9): une couche de surface de $5 \mathrm{~m}$ d'épaisseur, assez hétérogène, et une couche sous-jacente de sols organiques plus homogènes ;

- pour chacune des couches, on a déterminé la corrélation entre la cohésion non drainée $c_{u}$ et la résistance de pointe $\mathrm{q}$, et l'on a représenté sur l'écran la relation linéaire obtenue, l'intervalle de confiance de $E\left[c_{u}\right]$ pour un niveau de confiance de $\alpha=5 \%$ et l'intervalle de génération des valeurs de $c_{u}$ (qui tient compte de la variance des résidus et de l'intervalle de confiance sur $\left.E\left[c_{u}\right]\right)$. Ces courbes sont représentées sur la figure 12 ;

- à l'intérieur de chaque couche, on a utilisé ces corrélations pour compléter la description du site en générant des profils de cohésion non drainée, tous les $50 \mathrm{~cm}$ de profondeur, au droit des sections où l'on disposait des mesures pénétrométriques, et on a estimé l'incertitude correspondante. On a établi ainsi la carte des valeurs de $c_{\mathrm{u}}$ mesurées ou estimées par corrélation (fig. 13) ;

- l'étape suivante a consisté à générer les profils de cohésion non drainée pour toutes les sections de calcul, 


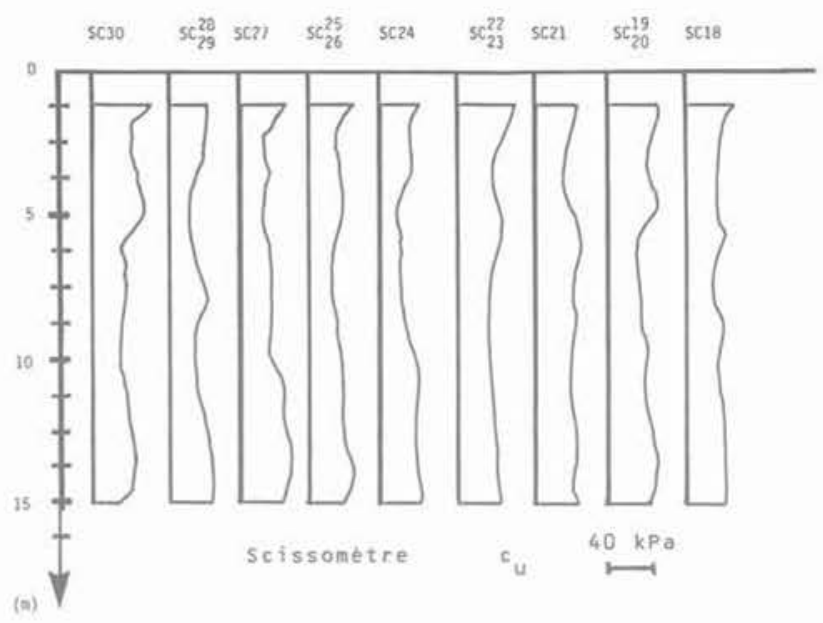

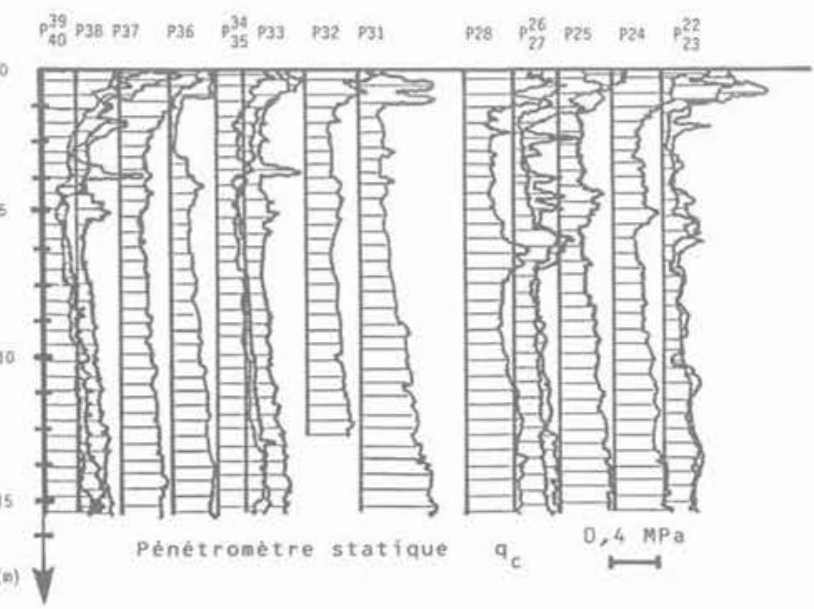

Fig. 11, - Carte des résultats de la reconnaissance géotechnique (scissomètre, pénétromètre statique).

Fig. 11. - Representation of the results of the geotechnical reconnaissance (vane test, static penetration test).

espacées de $50 \mathrm{~m}$. Les valeurs de la cohésion non drainée tous les $50 \mathrm{~cm}$ de profondeur ont êté obtenues par krigeage ponctuel, comme indiqué plus haut.

\subsection{Analyse de la stabilité}

L'analyse statistique des propriétés des sols ayant permis d'établir une carte des valeurs de la cohésion non drainée mesurées ou estimées, on a pu étudier l'incertitude sur la stabilité calculée du remblai sur une section de $550 \mathrm{~m}$ de longueur du tracé, en utilisant la procédure décrite précédemment. La longueur étudiée a été limitée à $550 \mathrm{~m}$ pour limiter loccupation de la mémoire de l'ordinateur. On a d'autre part modifié légèrement la structure spatiale des données, afin de restreindre le volume des calculs, tout en testant tous les aspects de la méthode d'analyse.

Dans la zone du tracé étudiée, le projet prévoyait la construction en deux phases d'un remblai de $8 \mathrm{~m}$ de hauteur, avec des banquettes latérales. Nous avons choisi pour simplifier d'étudier un remblai de $6 \mathrm{~m}$ de
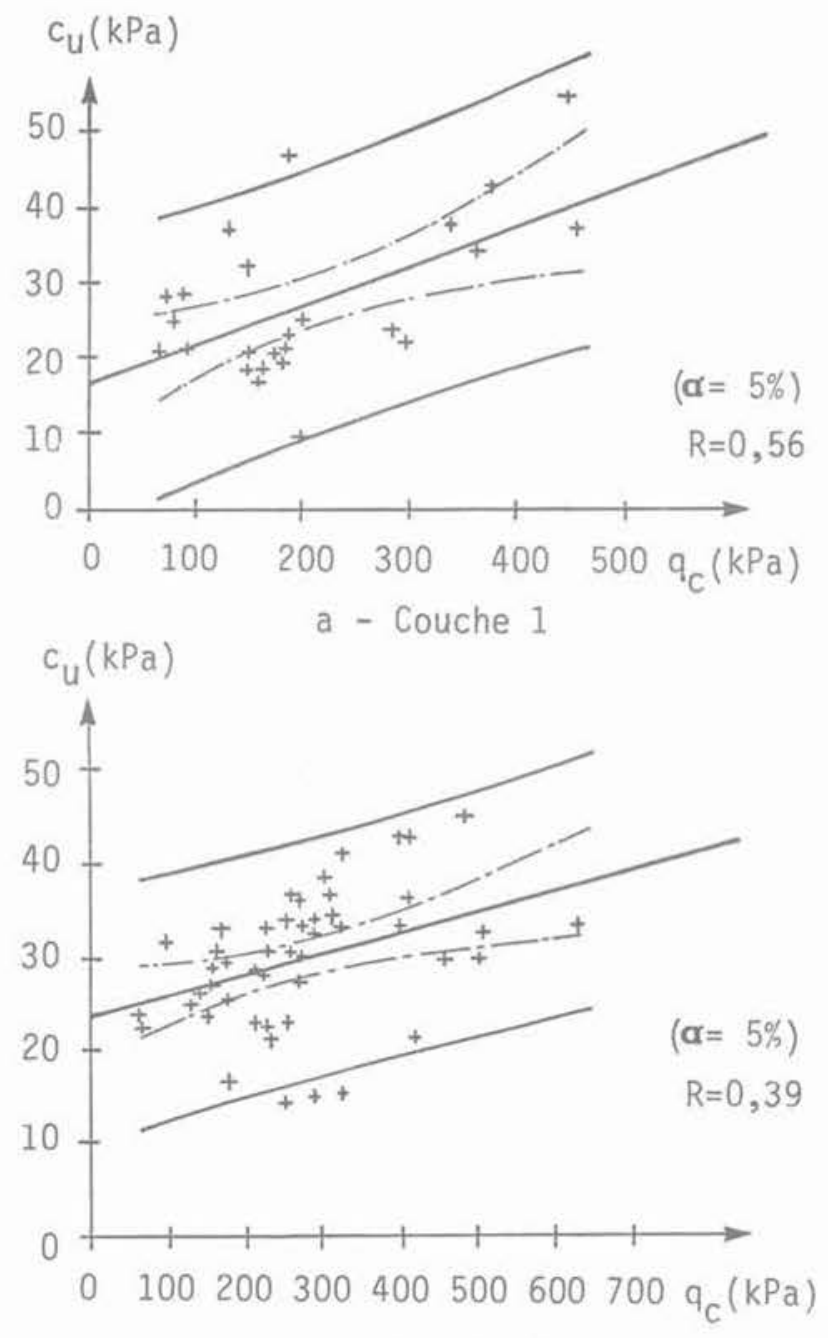

b - Couche 2

Fig. 12. - Corrélations entre $c_{u}$ et $q_{\varepsilon}$. Fig. 12. - Correlations between $c_{u}$ and $q_{c}$.
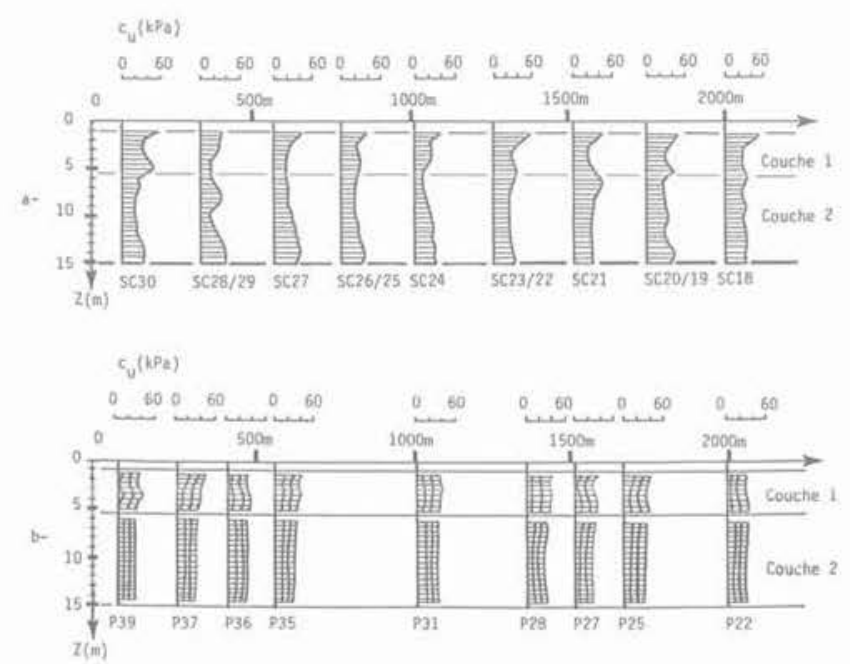

Fig. 13. - Valeurs de $c_{u}$ mesurées (a) et obtenues par corrélations (b).

Fig. 13. - $c_{\text {a }}$ values from vane tests (a) and from correlations (b) 
hauteur, sans banquettes latérales, construit en une seule phase sur une couche de sols mous d'épaisseur limitée à $8 \mathrm{~m}$. Ce remblai a les caractéristiques suivantes (fig. 14).

- largeur en tête $25 \mathrm{~m}$,

- largeur en pied $49 \mathrm{~m}$,

- pente des talus $1 / 2$,

- poids volumique $20 \mathrm{kN} / \mathrm{m}^{3}$.
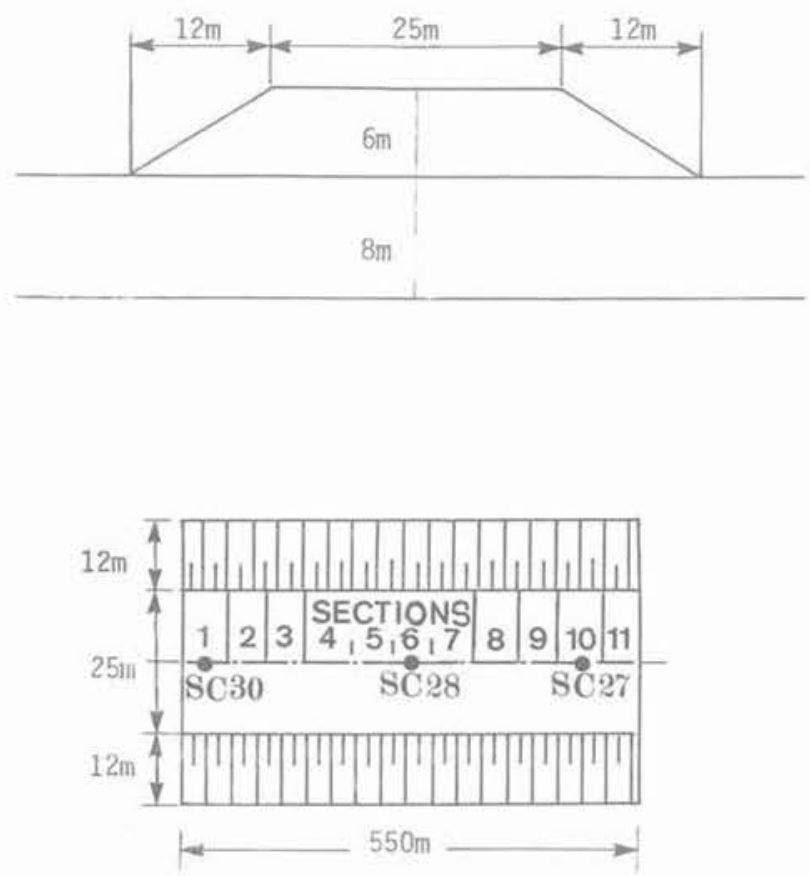

Fig. 14. - Geométrie du remblai et découpage pour le calcul.

Fig. 74. - Geometry of the fill and sections used in the analysis.

Le tronçon de remblai analysé a été découpé en 11 sections de $50 \mathrm{~m}$ de longueur (fig. 14). Les valeurs de la cohésion non drainée tous les $50 \mathrm{~cm}$ de profondeur dans chacune de ces sections sont représentées sur la figure 15. Ces profils de cohésion non drainée ont été estimés par krigeage. Dans les sections 1, 6 et 10 , on disposait de valeurs mesurées de la cohésion non drainée tous les mètres de profondeur. On observe que le krigeage a permis de retrouver ces points de mesure et de générer les valeurs intermédiaires, ainsi que leurs variances (la figure 15 représente les valeurs muyennes et les écarts types). Cependant, dans la croûte superficuelle, on ne disposait pas d'informations sur les valeurs de la cohésion non drainée et l'on a fait l'hypothèse que cette couche avait les mêmes propriétês qu'à un mètre de profondeur. D'autre part, compte tenu de la méthode de calcul utilisée, qui considère des couches de $50 \mathrm{~cm}$ d'épaisseur où la résistance et son ẻcart type sont constants, on a supposé le remblai construit après l'excavation d'une couche de $25 \mathrm{~cm}$ d'épaisseur, ce qui permet de ramener les mesures effectuées tous les mètres au milieu des couches du sol. frêquence

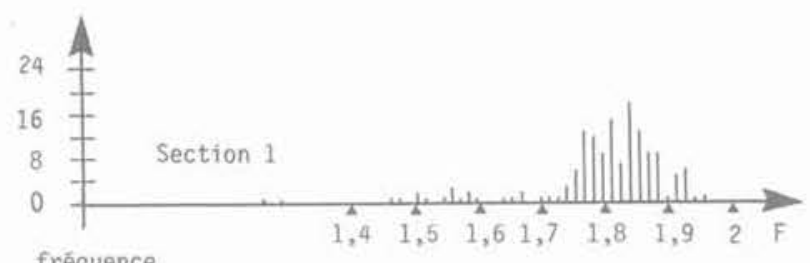

fréquence
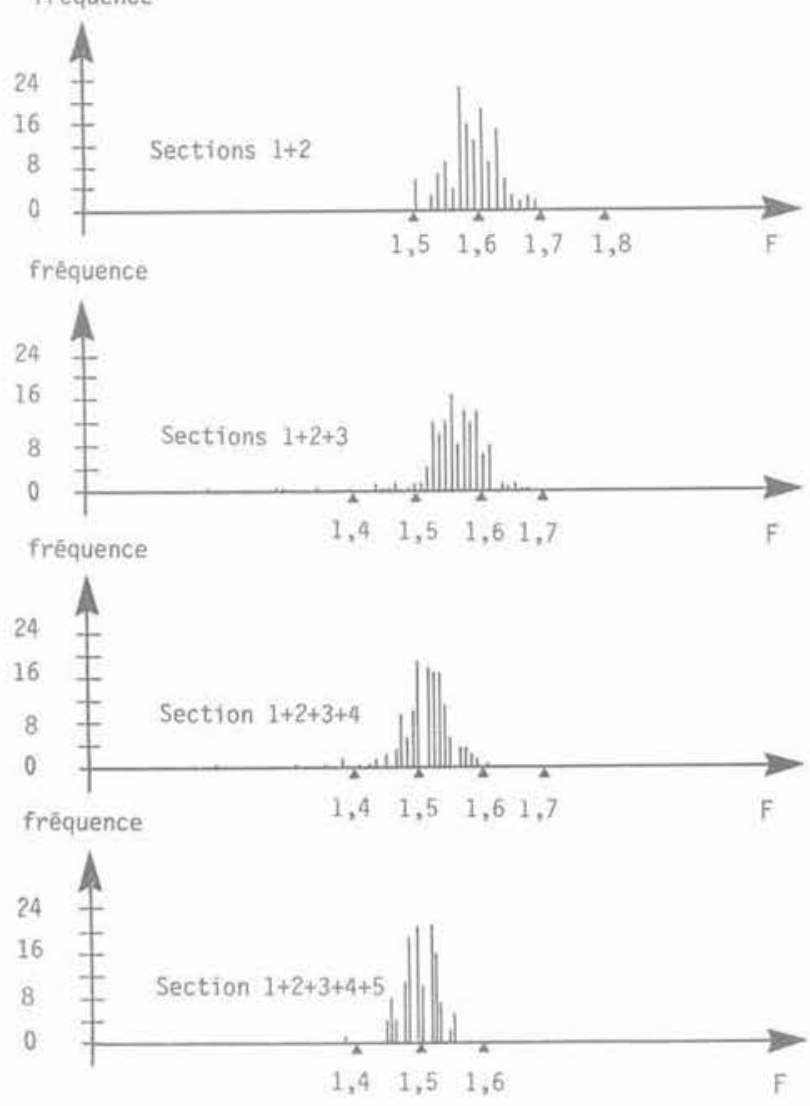

Fig. 16. - Histogrammes des coefficients de sécurité calculés.

Fig 16. - Histograms of the computed safety factors.

Pour l'analyse probabiliste de la stabilité du remblai, on a effectué 150 analyses de stabilité complètes de la zone étudiée, pour 150 ensembles de valeurs de la cohésion non drainée générées sur la base des valeurs moyennes et écarts types estimés par krigeage, pour une loi de distribution béta de paramètres $\alpha=1, \beta$ $=5$, avec un niveau de confiance de $95 \%$. Pour limiter l'occupation de la mémoire de l'ordinateur, on a décidé de limiter le balayage des tronçons et d'analyser la stabilité du remblai par zones constituées de cinq tronçons au plus. Malgré ces limitations, on a calculé 1745750 coefficients de sécurité, pour toutes les surfaces de rupture envisageables (axe du cylindre, rayon du cercle limitant le cylindre, longueur du cylindre). Ce chiffre élevé justifie a postériori les simplifications faites dans cette étude. Les valeurs minimales des coefficients de sécurité de chacun des 150 calculs ont été représentées sous forme d'histogrammes, pour les différentes longueurs et positions de la zone de rupture étudiées (figure 16). 


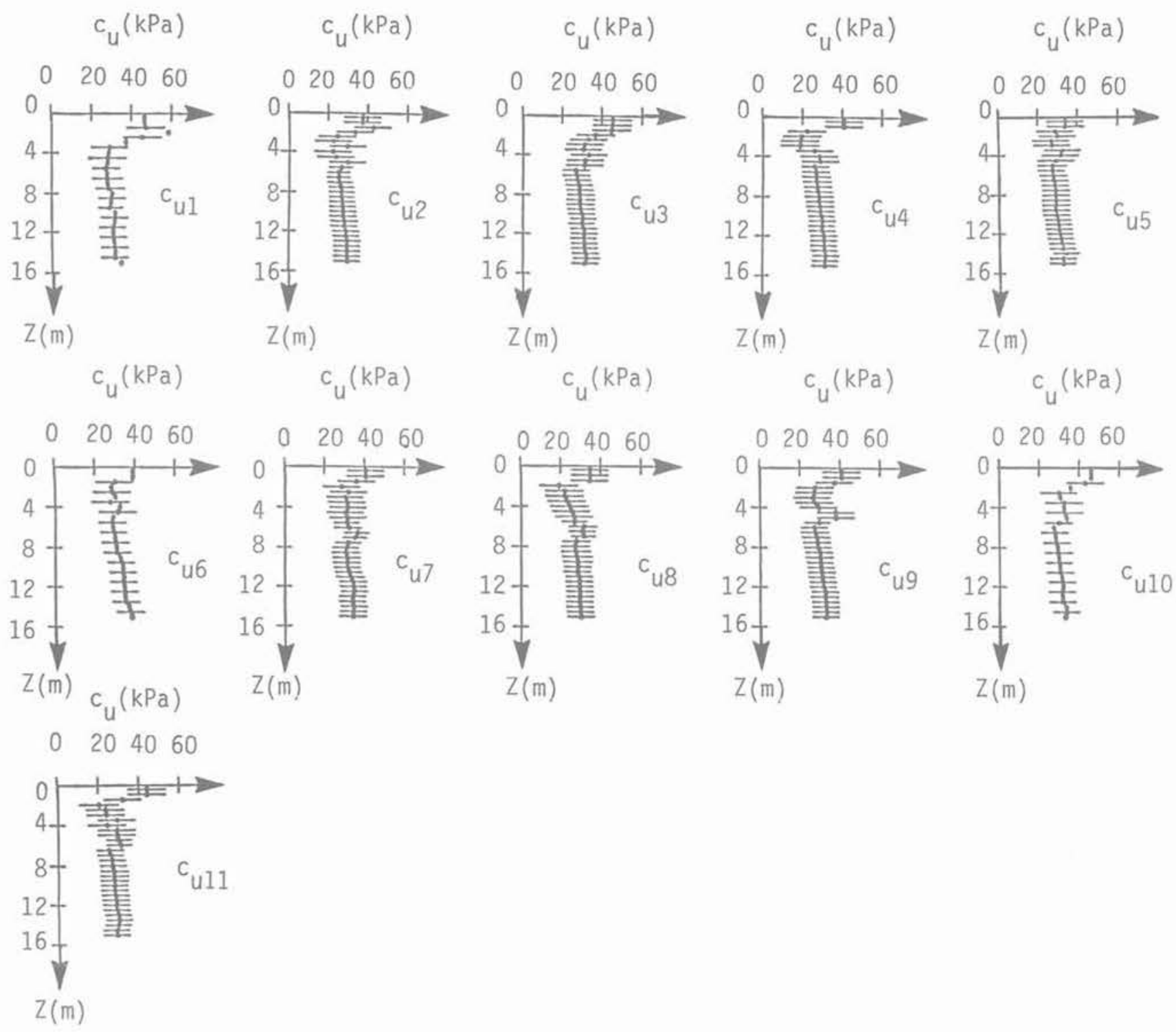

Fig. 15. - Données du calcul obtenues par krigeage.

Fig. 15 . - Design values obtained by kriging.

On a analysé ensuite les probabilités que le coefficient de sécurité calculé soit inférieur à quatre seuils $\beta=1,4-1,5-1,6-1,7$, pour chaque section ou combinaison de sections du remblai. Les résultats sont représentés sur la figure 17 , pour $\beta=1,5$. Cette figure fait ressortir des zones de rupture préférentielle, avec plusieurs maxima locaux. Néanmoins, il est impossible de tirer des conclusions plus précises sur la stabilité réelle de ce remblai car la méthode de calcul tridimensionnel utilisée n'a pas été testée sur des rup. tures de remblais réels et l'on ne connaît pas la valeur réelle du coefficient de sécurité à la rupture pour des cohésions non drainées mesurées au scissomètre sans correction en fonction de la plasticité du sol.

\section{COMMENTAIRES}

L'étude statistique et probabiliste décrite dans cet article a laissé à ses auteurs des impressions ambiguës, car elle montre à la fois que la détection des zones de plus grands risques le long d'un tracé de remblais sur sols mous est possible (ou qu'en tous cas il sera possible d'améliorer suffisamment les méthodes d'analyse pour pouvoir effectuer des analyses de risques sur une base statistique et probabiliste satisfaisante), mais aussi qu'il faut tant de données sur les propriétés mécaniques des sols du site, avec une structure spatiale adaptée, qu'il est peu probable que de telles données soient jamais disponibles en pratique. 


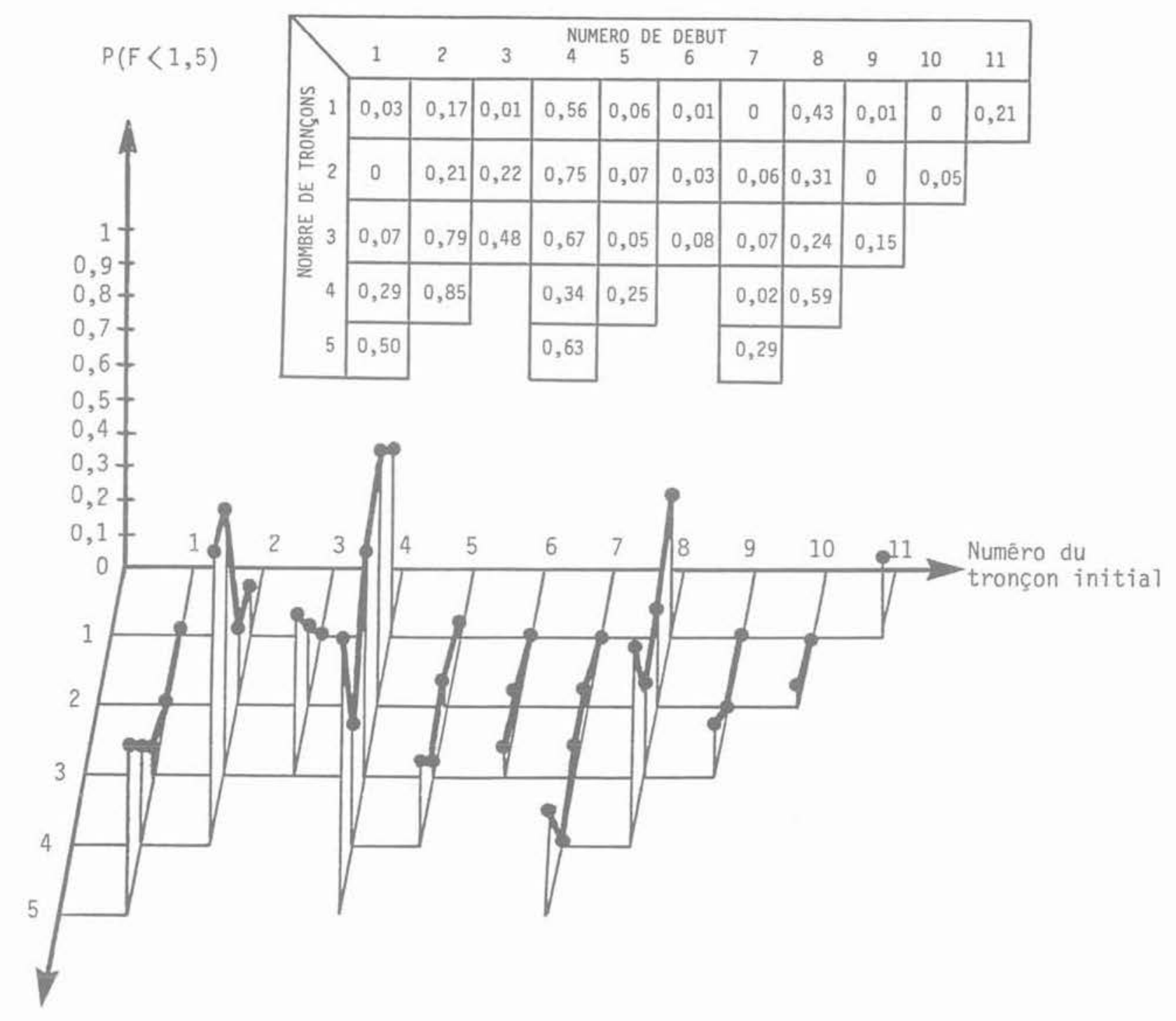

Nombre de

tronçons

Fig. 17. - Résultats de l'étude : Prob $[F<1,5]$.

Fig. 17. - Results of the analysis: Prob [F $<1.5]$.

Au plan des méthodes d'analyse statistique, la démarche adoptée est très simple, puisque l'on n'a utilisé que des régressions linéaires et du krigeage ponctuel. Il est possible que des techniques telles que le cokrigeage puissent améliorer la qualité de linterpolation des paramètres de calcul entre les points de mesure, lidéal étant que l'on puisse traiter simultanément les variations dans l'espace de toutes les caractéristiques mesurées du sol le long du tracé étudié... A notre connaissance, ces travaux restent à entreprendre. En fait, l'efficacité des analyses statistiques n'est pas uniquement liée aux méthodes d'analyse utilisées: les données disponibles doivent être adaptées à un traitement statistique des variations spatiales, ce qui n'était pas vraiment le cas sur le tracé étudié, malgré les apparences. Sur le tracé de l'autoroute A 64 dans les Barthes de l'Adour étudié en 1978, la reconnaissance avait été structurée de façon traditionnelle, en répartissant les sondages au scissomètre de façon régulière, pour avoir une idée des variations de la cohésion non drainée sur l'axe du tracé et sous les bords du remblai, les sondages au pénétromètre venant confirmer l'homogénéité des sols entre les sondages au scissomètre. Cette implantation des sondages a gêné l'établissement des corrélations car il a fallu considérer comme voisins des sondages distants de 10 à $20 \mathrm{~m}$; la mauvaise qualité des corrélations provient sans doute pour partie de l'espacement des mesures corrélées. La réalisation d'un certain nombre de sondages voisins pour établir les corrélations entre paramètres peut être conseillée si 
l'on veut appliquer efficacement cette méthode d'analyse sur d'autres sites. D'autre part, il serait intéressant d'étudier également les variations des propriétés des sols perpendiculairement à l'axe du tracé, puisque les remblais autoroutiers ont couramment des largeurs à la base de lordre de $50 \mathrm{~m}$.

Un autre aspect important de cette analyse statistique est le volume considérable des données qu'il faut manipuler et stocker simultanément dans la mémoire de l'ordinateur. Cette étude a été réalisée sur l'ordinateur DPS8 du Centre Informatique Recherche d'Arcueil, commun à l'INRETS et au LCPC, et les seules contraintes subies étaient liées aux procédures d'exploitations courantes de l'ordinateur, mais les besoins de cette étude pouvaient dépasser ces limites, pour peu que l'on ait décidé de traiter une zone plus longue du tracé. Le relevé manuel des enregistrements des sondages pénétrométriques est pour sa part une tâche fastidieuse, qu'il faudrait pouvoir supprimer si cette procédure d'analyse devait se répandre.

Au plus de l'analyse probabiliste, l'utilisation de la méthode de Monte Carlo nécessite un très grand nombre de calculs, compte tenu de la méthode classiquement utilisée pour analyser la stabilité des remblais sur sols mous. Une autre difficulté est celle du biais de cette méthode d'analyse, qui est mal connu dans le cas de zones de rupture de longueur finie. Le schéma de calcul utilisé est très simple et il mériterait certaines améliorations, par exemple pour la géométrie de la surface de rupture. Néanmoins, il faut garder à l'esprit que cette forme de cylindre à extrémités planes a permis de réaliser une analyse discrète du tracé et que, si une autre géométrie de rupture est adoptée, il faudra adapter la description du sol et le balayage du tracé à cette nouvelle méthode d'analyse. Tout cela demande un travail important, dont l'utilité pratique n'est pas assurée.

On peut penser qu'une approche différente, qui considèrerait le calcul du moment résistant sur un cylindre de rupture comme un processus d'homogénéisation des valeurs de la cohésion non drainée sur cette surface de rupture, avec une valeur moyenne et un écart type dépendant du volume de sol concerné, serait d'utilisation plus simple. C'est ainsi qu'avait raisonné VAN. MARCKE (1977) pour l'analyse de la rupture d'un remblai à New Liskeard. Il a calculé une longueur critique de rupture de ce remblai en utilisant une surface de rupture cylindrique à extrémités planes semblables à celle de la présente étude, mais son étude suppose que le sol du site analysé est statistiquement homogène. en ce sens que la position des points de mesure n'a pas d'influence sur la probabilité des valeurs mesurées. Une telle approche reste insuffisante pour traiter des problèmes réels et il faudrait définir des propriétés statistiques des moyennes variables en fonction de la position de la surface de rupture dans l'espace. De telles recherches mériteraient d'être entreprises.

\section{CONCLUSION}

La procédure d'évaluation de la probabilité de rupture des remblais de grande longueur édifiés sur des sols mous qui a été présentée dans cet article comporte deux phases successives:

- on commence par analyser l'information fournie par la reconnaissance géotechnique du site, en utilisant les corrélations locales (à l'échelle du site) existant entre les paramètres pour compléter la carte des valeurs mesurées des paramètres directement utilisés pour les calculs. Puis, compte tenu de la méthode de calcul que l'on a prévu d'utiliser, on interpole les valeurs connues des paramètres pour obtenir les valeurs de ces paramètres aux points où l'on en a besoin pour les calculs. Cette interpolation entre des valeurs connues avec une certaine marge d'incertitude est effectuée par krigeage. Cette premiere phase de l'analyse produit un tableau de valeurs de la moyenne et de l'écart type des para. mètres du calcul en tous les points utiles ;

- la seconde phase de l'analyse consiste à évaluer la probabilité de rupture du remblai sur la base des valeurs des paramètres de calcul déduites de la première partie de l'étude. En faisant varier la position de la surface de rupture potentielle le long du tracé, on peut déterminer la zone où la stabilité du remblai est la plus critique et réagir en conséquence.

Les résultats présentés ici ne constituent qu'une première étape vers une procédure d'analyse des risques éventuellement utilisable en pratique. Parmi les améliorations qui pourraient lui être apportées, on peut citer l'utilisation des techniques de cokrigeage ou des corrélations multiples pour l'analyse des propriétés géo. techniques des sols, la prise en compte de variations transversales des propriétés des sols par rapport à l'axe du tracé et une meilleure description des surfaces de rupture potentielles. Mais l'utilisation éventuelle d'une telle méthode d'analyse des risques dans des projets réels nécessite une réflexion approfondie sur la consistance et la structure spatiale des reconnaissances géotechniques pour les études de tracés, en tenant compte des contraintes budgétaires inévitables.

\section{BIBLIOGRAPHIE}

ALONSO E.E. (1976), Risk analysis of slopes and its application to slopes in Canadian sensitive clays. Géotechnique, vol. 26, n³, pages 453-472.

BAGHERY S. (1980), Probabilités et statistiques en mécanique des sols. Analyse probabiliste de la stabilité et des tassements de remblais sur sols compressibles (site expérimental de Cubzac-lesPonts). Thèse de docteur-ingénieur, Ecole Nationale des Ponts-et-Chaussées, Paris, 184 pages.

BAGHERY S., MAGNAN J.P. (1983), Analyse probabiliste de la stabilité et des tassements des remblais du site expérimental de Cubzac-les-Ponts. Laboratoire Central des Ponts et Chaussées, Paris, Rapport de recherche LPC $n^{\circ} 122,71$ pages.

BENJAMIN J.R., CORNELL C.A. (1970), Probability, statistics and decision for civil engineers. MacGrawHill, New-York.

BJERRUM L. (1972), Embankments on soft ground General report. Proc., ASCE Specialty Conference on Earth - and Earth-supported Structures, Purdue Univ., LaFayette, Ind., vol. 2, pages 1-52. 
DELFINER P. (1979), Basic introduction to geostatistics. Centre de Géostatistique et de Morphologie Mathématique, Ecole des Mines de Paris, Fontainebleau.

GUILLAUME A. (1977), Analyse des variables régionalisées. Traitement du signal en sciences de la terre. Doin, Paris.

JOURNEL A.G. (1977), Géostatistique minière. Centre de Géostatistique, Ecole des Mines de Paris, Fontainebleau.

LEBART L., FENELON J.P. (1977), Statistiques et informatique appliquées. Dunod, Paris, $2^{e}$ édition.

LEROUEIL S., MAGNAN J.P., TAVENAS F. (1985), Remblais sur argiles molles. Technique et Documentation, Lavoisier, Paris, 342 pages.

MAGNAN J.P. (1982), Les méthodes statistiques et probabilistes en mécanique des sols. Presses de I'Ecole Nationale des Ponts et Chaussées, Paris, 203 pages.

MAGNAN J.P. et al. (1984), Remblais et fondations sur sols compressibles. Presses de l'Ecole Nationale des Ponts et Chaussées, Paris, 253 pages.
MAGNAN J.P., BAGHERY S. (1982), Statistiques et probabilités en mécanique des sols. Etat des connaissances. Laboratoire Central des Ponts et Chaussées, Paris, Rapport de recherche LPC n 109,192 pages.

MAGNAN J.P., BRU J.P., PEIGNAUD M. (1980), Quelques règles pour la reconnaissance géotechnique des sites compressibles. Bulletin de liaison des Laboratoires des Ponts et Chaussées, Paris, $\mathrm{n}^{\circ} 108$, pages $5-12$.

MAGNAN J.P., BRU J.P., PILOT G. (1987), Remblais d'essai pour un tracé routier sur sol mou. Comptes rendus, $9^{\circ}$ Congrès Européen de Mécanique des Sols et des Travaux de Fondations, Dublin, A.A. Balkema, Rotterdam, vol. 1, pages 71-74.

MAHDAVI C. (1985), Analyse probabiliste du comportement des sols et des ouvrages. Evaluation des risques dans les études géotechniques de tracés de remblais sur sols mous. Thèse de docteur-ingénieur, Ecole Nationale des Ponts et Chaussées, Paris, 180 pages.

VANMARCKE E.H. (1977), Reliability of earch slopes. ASCE, Journal of Geotechnical Engineering Division, vol. $110, \mathrm{n}^{\circ} 2$, pp. 145-188. 